\title{
AVAliaÇão da AgITAÇÃo E dA AERAÇÃo NA PRODUÇÃo de $\beta$ - GALACTOSIDASE POR Kluyveromyces marxianus CBS 6556 A PARTIR DE SORO DE QUEIJO
}

\author{
B. L. B. PERINI ${ }^{1}$, H. C. M. SOUZA ${ }^{3}$ e A. L. S. SCHNEIDER ${ }^{1,2}$ \\ ${ }^{1}$ Universidade da Região de Joinville, Mestrado em Engenharia de Processos \\ ${ }^{2}$ Universidade da Região de Joinville, Departamento de Engenharia Química \\ ${ }^{3}$ Universidade da Região de Joinville, Departamento de Engenharia Ambiental \\ E-mail para contato: andrea.lima@univille.net
}

\begin{abstract}
RESUMO -A intolerância à lactose está associada à deficiência na produção da enzima lactase, sendo que, uma alternativa às pessoas com esta deficiência é a ingestão de suplementos de lactase. A partir de resultados preliminares de um planejamento experimental (DCCR Delineamento Composto Central Rotacional), para avaliar as melhores condições na produção da enzima $\beta$-galactosidase de Kluyveromyces marxianus CBS 6556, com variáveis: soro de queijo, milhocina e temperatura, foi possível determinar a melhor condição para produção da lactase. Com base nos resultados do DCCR, para avaliar a influência da agitação e da aeração na produção da enzima por cultivo submerso, duas condições em duplicata foram conduzidas em fermentador Biostat B. As condições estudadas foram $200 \mathrm{~min}^{-1}$ e 1,33 vvm $\left(\mathrm{K}_{\mathrm{L}} \mathrm{a}=19 \mathrm{~h}^{-1}\right)$ e 400 $\min ^{-1}$ e $2,7 \mathrm{vvm}\left(\mathrm{K}_{\mathrm{L}} \mathrm{a}=19 \mathrm{~h}^{-1}\right)$, sendo que os valores de atividade enzimática alcançados foram $4,53 \pm 0,46 \mathrm{U} \mathrm{mL}^{-1}$ e $6,59 \pm 0,20 \mathrm{U} \mathrm{mL}^{-1}$, respectivamente.
\end{abstract}

\section{INTRODUÇÃO}

A lactose, açúcar presente no leite e seus derivados, é hidrolisada pela ação da enzima $\beta$-galactosidase gerando moléculas menores, a glicose e a galactose. A deficiência na produção da $\beta$-galactosidase pode ocasionar a ocorrência de uma síndrome conhecida como intolerância à lactose, sendo que as pessoas que apresentam este quadro não conseguem hidrolisá-la.

A enzima $\beta$-galactosidase (EC 3.2.1.23) encontra-se entre as enzimas de grande interesse industrial empregada na hidrólise da lactose de leites e soro de queijo, obtendo, assim, alimentos com baixos teores de lactose, cujo resultado é a melhora da solubilidade e da digestibilidade de leites e derivados lácteos, tornando-os ideais a consumidores intolerantes a este açúcar (MANERA et al., 2011).

Diferentes processos industriais produzem resíduos que necessitam de destino adequado, sendo porém, na maioria das vezes, descartados sem nenhum tratamento prévio em corpos d'água (rios, lagos, mares), acarretando morte dos peixes e outros organismos aquáticos, comprometendo ainda a estrutura físicoquímica dos solos e acentuando a poluição ambiental (TONI et al., 2012).Um exemplo de resíduo é o soro de queijo, que vem sendo testado em diversos estudos a fim de aproveitar o alto teor de lactose e ainda diminuir o seu impacto quando lançado como efluente. Este substrato tem sido utilizado em processos fermentativos para produção de compostos orgânicos e enzimas, como a $\beta$ galactosidase.

Considerando esta situação, este trabalho buscou avaliar os efeitos da agitação e da aeração para a 
produção da enzima $\beta$-galactosidase de Kluyveromyces marxianus CBS 6556 em biorreator, a partir de fontes alternativas de carbono e nitrogênio.

\section{METODOLOGIA}

\subsection{Micro-organismo eOutrasCondições}

O micro-organismo utilizado neste trabalho foi a levedura Kluyveromyces marxianus CBS 6556. Para manutenção da cepa utilizou-se o cultivo em placas de Petri com o meio YPD (extrato de levedura 1\%, peptona $1 \%$, glicose $2 \%$ e ágar) com repiques mensais e mantidos em refrigerador a $4{ }^{\circ} \mathrm{C}$. A ativação foi feita à temperatura de $37^{\circ} \mathrm{C}$, em frascos de Erlenmeyer não aletados $(1000 \mathrm{~mL})$, contendo $300 \mathrm{~mL}$ de meio YPD (extrato de levedura, peptona e glicose), em agitação circular de $180 \mathrm{~min}^{-1}$ por aproximadamente 12 e 15 horas a $30^{\circ} \mathrm{C}$. A fração de inoculo foi de $10 \%$.

Os substratos utilizados foram soro de queijo, fornecido pela Indústria Victória e Milhocina, fornecido pela Corn Products. O soro de queijo foi desproteinizado para facilitar posteriormente a leitura de absorbância.

\subsection{Condições de Cultivo em Biorreator}

Os ensaios em biorreator foram realizados em duplicatas, considerando o volume útil de 1,5 $\mathrm{L}$ sendo $10 \%(\mathrm{~V} / \mathrm{V})$ de inóculo, com a utilização de $1350 \mathrm{~mL}$ de soro de queijo (concentração de $900 \mathrm{~mL} \mathrm{~L}^{-1}$, equivalente à $25 \mathrm{~g} \mathrm{~L}^{-1}$ de lactose) e $27 \mathrm{~g}$ de milhocina (concentração de $18 \mathrm{~g} \mathrm{~L}^{-1}$ ). A temperatura de operação foi de $31{ }^{\circ} \mathrm{C}$, com duração de $24 \mathrm{~h}$ e intervalos de amostragem realizados nos seguintes tempos: $0,3,6$, 9, 14, 19, $24 \mathrm{~h}$. Duas condições de cultivo foram pré-estabelecidas com diferentes valores de agitação e aeração, sendo que a condição (a) (200 $\left.\min ^{-1} ; 2 \mathrm{~L} \mathrm{~min}^{-1} \mathrm{e} 1,33 \mathrm{vvm}\right)$, resultou em um valor de $\mathrm{K}_{\mathrm{L}} \mathrm{a}=19 \mathrm{~h}^{-1}$, e a condição (b) $\left(400 \mathrm{~min}^{-1} ; 4 \mathrm{~L} \mathrm{~min}^{-1}\right.$ e 2,67 vvm), resultou em um valor de $\mathrm{K}_{\mathrm{L}} \mathrm{a}=53 \mathrm{~h}^{-1}$. A fim de evitar a formação de espuma, apenas na condição (b) foi utilizado $0,2 \mathrm{~mL}$ polipropilenoglicol 2000 (Fluka).

\section{RESULTADOS E DISCUSSÃO}

Na Figura 1 está demonstrada a produção da lactase por Kluyveromyces marxianus CBS 6556 com monitoramento do oxigênio dissolvido, atividade enzimática, consumo de lactose, produção de biomassa e produção de etanol, durante os cultivos realizados no biorreator para a condição (a) $200 \mathrm{~min}^{-1} / 2 \mathrm{~L} \mathrm{~min}^{-1} \mathrm{e}$ $\mathrm{K}_{\mathrm{L}}$ a de $19 \mathrm{~h}^{-1}$. 


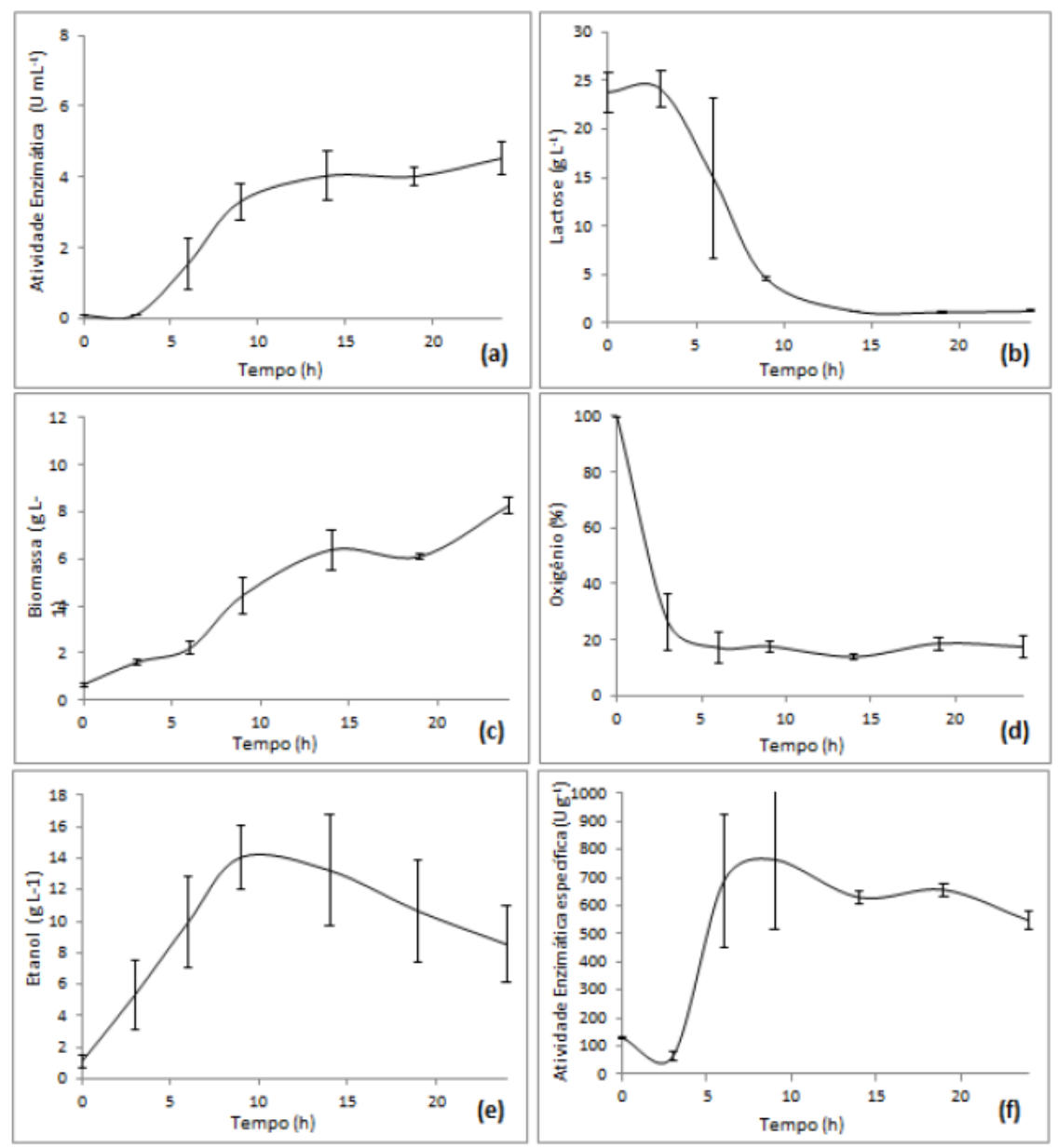

Figura 1 -Acompanhamento da produção de $\beta$-galactosidase para condição $200 \mathrm{~min}^{-1}$ e $2 \mathrm{~L} \mathrm{~min}^{-1}$ para atividade enzimática volumétrica (a), consumo de lactose (b), produção de biomassa (c), oxigênio dissolvido (d), produção de etanol (e) e atividade enzimática específica (f).

De modo geral, pode-se verificar que o consumo de substrato foi completo após 14 horas de cultivo, sendo que a partir deste tempo não houve mais produção de $\beta$-galactosidase, alcançado a valor máximo de 4,53 $\pm 0,46 \mathrm{U} \mathrm{mL}^{-1}$ em 24 horas de cultivo. Neste mesmo tempo, a quantidade máxima de biomassa, 8,25 \pm $0,35 \mathrm{~g} \mathrm{~L}^{-1}$ foi produzida, enquanto que a quantidade de oxigênio dissolvido disponível no meio reduziu-se consideravelmente até o tempo de 3 horas de cultivo, mantendo-se constante até o fim do cultivo.

Esta limitação de oxigênio no meio de cultivo, favoreceu o metabolismo anaeróbio da levedura levando à produção de etanol. Assim como observado por outros autores, devido a presença de álcool desidrogenase, após algumas horas de cultivo, o etanol passou a ser consumido, contribuindo, para a elevação da quantidade de biomassa produzida.

Na Figura 2 está demonstrada a produção da lactase por Kluyveromyces marxianus CBS 6556 para a condição (b) $400 \mathrm{~min}^{-1} / 4 \mathrm{~L} \mathrm{~min}^{-1}$ e $\mathrm{K}_{\mathrm{L}}$ a de $53 \mathrm{~h}^{-1}$. 

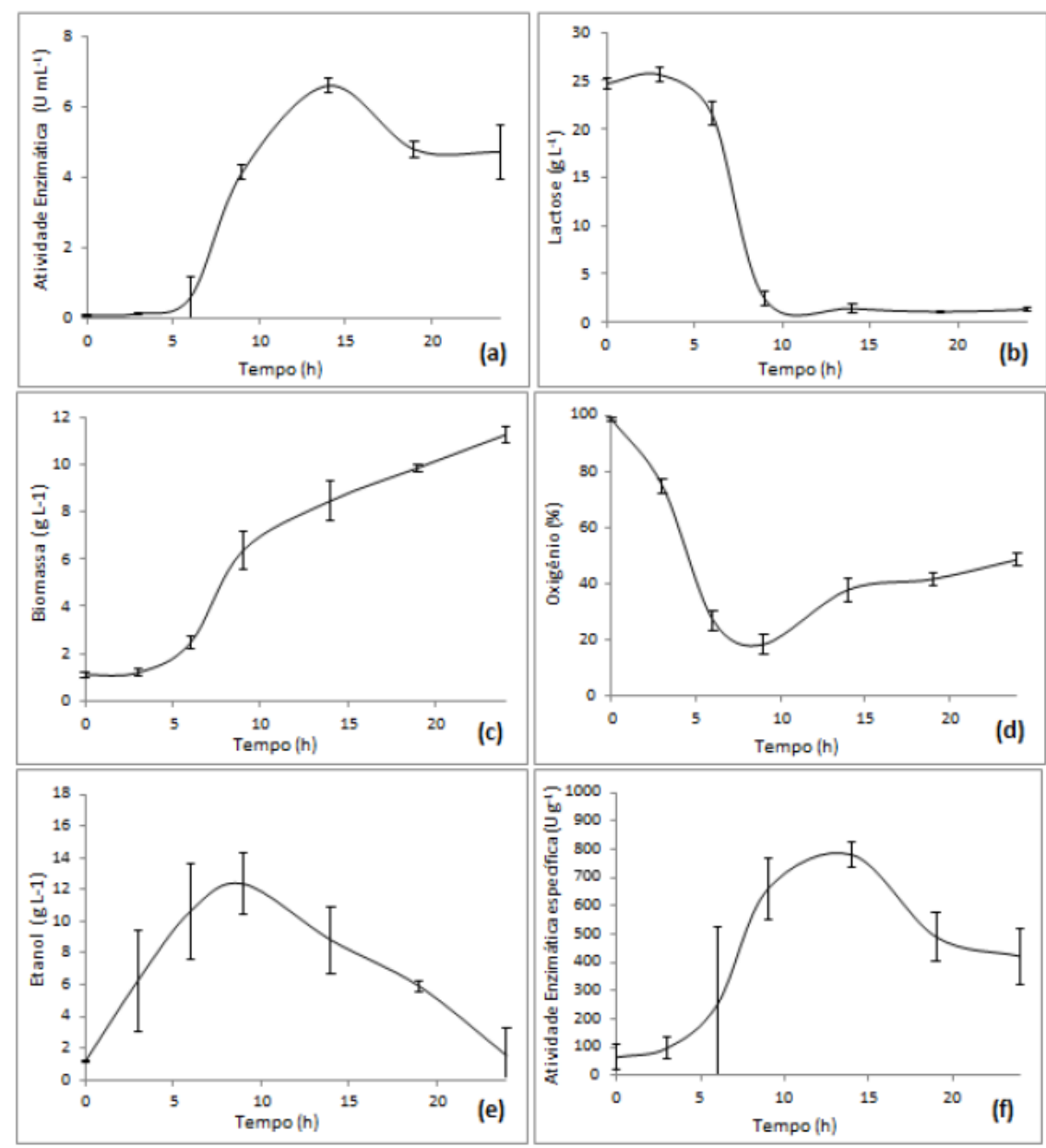

Figura 2 -Acompanhamento da produção de $\beta$-galactosidase para condição $400 \mathrm{~min}^{-1}$ e $4 \mathrm{~L} \mathrm{~min}^{-1}$ para atividade enzimática volumétrica (a), consumo de lactose (b), produção de biomassa (c), oxigênio dissolvido (d), produção de etanol (e) e atividade enzimática específica (f).

A atividade enzimática máxima foi encontrada no tempo de 14 horas de cultivo, alcançando 6,59 $\pm 0,20 \mathrm{U} \mathrm{mL}^{-1}$,enquanto que a produção de biomassa foi observada até o fim do cultivo, em virtude do consumo de etanol, tal como observado na condição anterior. No entanto, devido a maior condição de oxigenação nesta condição, houve formação de menor quantidade de etanol permitindo seu consumo na sua totalidade em 24 horas de cultivo. Pode-se observar que a lactose foi praticamente consumida por completo até as 9 horas de cultivo, sendo acompanhada por uma ligeira queda no teor de oxigênio disponível no meio até $20 \%$ estabilizando em seguida em $40 \%$ de $\mathrm{O}_{2}$ após as 14 horas até o término do cultivo.

Rech et al. (1999) ao avaliar a produção da lactase pela mesma linhagem de $K$. marxianus em biorreator, a partir de $50 \mathrm{~g} \mathrm{~L}^{-1}$ de lactose de soro de queijo em condições de agitação e aeração de 500 $\min ^{-1}, 3 \mathrm{~L} \mathrm{~min}^{-1}$ e $2 \mathrm{vvm}$, obteve fator de conversão de substrato em células $\left(\mathrm{Y}_{\mathrm{X} / \mathrm{S}}\right)$ de $0,287 \mathrm{~g} \mathrm{~g}^{-1}$, fator de conversão de substrato em enzima ( $\left.\mathrm{Y}_{\mathrm{P} / \mathrm{S}}\right)$ de $129,7 \mathrm{U} \mathrm{g}^{-1}$ e atividade enzimática de $5 \mathrm{U} \mathrm{mL}^{-1}$. $\mathrm{Na}$ condição (b), a partir da metade $\left(25 \mathrm{~g} \mathrm{~L}^{-1}\right)$ da concentração de lactose contidas em soro de queijo, podese observar um fator de conversão de substrato em enzima duas vezes maior, de $266,7 \mathrm{U} \mathrm{g}^{-1}$, enquanto 
que o fator de conversão de substrato em células e a atividade enzimática foram de $0,300 \mathrm{~g} \mathrm{~g}^{-1}$ e $6,59 \pm$ $0,20 \mathrm{U} \mathrm{mL}^{-1}$.

Outros autores avaliaram os efeitos da agitação e aeração a fim de maximizar a produção de $\beta$ galactosidase de $K$. marxianus em biorreator, sob uma quantidade inicial de lactose, muito próxima à deste trabalho, contida no meio de cultivo (ALVES et al., 2010). No entanto, estes autores utilizaram lactose PA (28,8 $\left.\mathrm{g} \mathrm{L} \mathrm{L}^{-1}\right)$ como fonte de carbono, em conjunto de $\left(\mathrm{NH}_{4}\right) \mathrm{SO}_{4}$ e extrato de levedura como fonte de nitrogênio, enquanto que neste trabalho, o meio de cultivo foi elaborado apenas pelos resíduos agroindustriais, soro de queijo, tendo a lactose como fonte de carbono $\left(25 \mathrm{~g} \mathrm{~L}^{-1}\right)$ e a milhocina, como fonte de nitrogênio.

Nestes ensaios em biorreator, esperava-se alcançar, pelo menos, valores de atividade enzimática volumétrica próximos ao valor previsto pelo modelo gerado no planejamento experimental DCCR realizado em shaker (PERINI, 2013), que prevê o valor da atividade enzimática volumétrica de 7,78 $\mathrm{U} \mathrm{mL}^{-1}$. Os resultados obtidos nos ensaios realizados forneceram os valores de 6,59 $\pm 0,20 \mathrm{U} \mathrm{mL}^{-1}$ para $\mathrm{K}_{\mathrm{L}}$ a de $53 \mathrm{~h}^{-1} \mathrm{e}$ 4,53 $\pm 0,46 \mathrm{U} \mathrm{mL}^{-1}$ para $\mathrm{K}_{\mathrm{L}}$ a de $19 \mathrm{~h}^{-1}$. A diferença do valor previsto pelo modelo foi de $25,3 \%$ para a condição (a) e 15,3\% para a condição (b), indicando que a condição (b) em biorreator se aproximou mais das condições de agitação e aeração dos experimentos em shaker, que geraram o modelo.

\section{CONCLUSÕES}

Na condição de $900 \mathrm{~mL} \mathrm{~L}^{-1}$ de soro de queijo, $18 \mathrm{~g} \mathrm{~L}^{-1}$ de milhocina e a $31{ }^{\circ} \mathrm{C}$, avaliou-se duas condições de agitação e aeração diferentes: uma mais branda $\left(K_{L} a=19 h^{-1}\right)$ e outra mais severa $\left(K_{L} a=\right.$ $\left.53 \mathrm{~h}^{-1}\right)$, sendo que a melhor condição estabelecida para a produção da $\beta$-galactosidase proveniente de Kluyveromyces marxianus CBS 6556 com soro de queijo e milhocina foi com agitação de $400 \mathrm{rpm}$ e aeração de 2,67 vvm, alcançando uma atividade enzimática de 6,59 $\pm 0,20 \mathrm{U} \mathrm{mL}^{-1}$ em 14 horas de cultivo. Em ambas as condições, foi observada a produção de etanol até as 9 horas de cultivo, seguido do consumo do mesmo até o fim dos processos.Os resultados deste trabalho mostram uma possibilidade para o cultivo de Kluyveromyces marxianus CBS 6556 visando a produção da enzima $\beta$-galactosidase utilizando resíduos agroindustriais (soro de queijo e milhocina) em biorreator.

\section{REFERÊNCIAS}

ALVES, F. G.; FILHO, F. M.; BURKERT, J. F. M.; KALIL, S. J. Maximization of $\beta$-galactosidase production: a simultaneous investigation of agitation and aeration effects. Appl. Biochemical Biotechnological, v. 160, p. 1528-1539, 2010.

MANERA, A. P.; ORES, J. da C.; RIBEIRO, V. A.; RODRIGUES, M. I.; KALIL, S. J.; MAUGERI FILHO, F. Utilização de resíduos agroindustriais em processo biotecnológico para produção de $\beta$-galactosidase de Kluyveromyces marxianus CCT 7082 . Acta Scientarium Technology, v. 33, n. 2, p. 155-161, 2011.

PERINI, B. L. B. Produção de $\beta$-galactosidase comKluyveromyces marxianus CBS 6556 a partir de soro de queijo. Dissertação de Mestrado em Engenharia de Processos. Universidade da Região de Joinville, Joinville, 2013.

PERINI, B.L.B.; SOUZA, A.C.M; KELBERT, M.; APATI, G.P.; PEZZIN, A.P.T.; SCHNEIDER, A.L.S. Production of $\beta$-galactosidase from cheese whey using Kluyveromyces marxianus CBS 6556. Chemical Engineering Transcations, v. 32, p.991-996, 2013.

RECH, R.; CASSINI, C. F.; AYUB, M. A. Z. Utilization of protein-hydrolyzed cheese whey for production of $\beta$-galactosidase by Kluyveromycesmarxianus.Journal of Industrial Microbiology \& Biotechnology, v. 23, p. 91-96, 1999.

TONI, J. C. V.; IMAMURA, K. B.; DORTA, C. Reaproveitamento do soro de leite bovino para produção de biomassa por Kluyveromyces marxianus 229. Revista Alimentus v. 2, p.36-57, 2012. 